\title{
Role of L. reuteri in Maintenance and Genesis of GI Issues
}

\section{Ridhima Bhalla*}

Post Graduate, Public Health and Nutrition, Senior Manager-Scientific Services (McCann Health, India)

*Corresponding Author: Post Graduate, Public Health and Nutrition, Senior Manager-Scientific Services (McCann Health, India).

Received: August 21, 2019; Published: September 27, 2019

\section{Gut Microbiota and its association with FGIDs}

The first two years of life are an important part of this period, which is characterized by accelerated growth rate and development of several organs and systems. Several gastrointestinal discomfort signs and symptoms can be observed in infants, which may be linked to several anatomical and functional changes observed at this stage of life [1]. Therefore, a Functional Gastrointestinal Disorder (FGID) is a physiological outcome of altered microbiota, physiological and functional immaturity and other environmental factors including diet [7].

The occurrence of FGIDs is common in infancy, such as regurgitation, colic, functional constipation, functional diarrhea etc. Many of these symptoms may be transient and are attributed to physiological and functional immaturity, dysbiosis and/or are considered as part of the development of the gastrointestinal tract [6]. The gut microbiota is a key player in determining gut health and function. Alterations in the gut microbiota composition have been well described in several functional gastrointestinal disorders and are reviewed exhaustively by the Rome Working Team in Rome III criteria [2]

\section{L. reuteri - gut friendly bacteria}

L. reuteri is found naturally in the breast milk, which is demonstrated to have probiotic properties [3].
Various studies have proved that L. reuteri improves the composition of the gut microbiota. As compared with placebo, L. reuteri promotes gut health through a reduction of $E$. coli colonization [5]. Therefore, breastfeeding becomes the way to ensure that an infant receives beneficial microbiota and optimal substrates for the growth of these bacteria [3]

L. reuteri strains can positively influence human health as it produce reuttering, a broad-spectrum antibacterial substance, which is capable of inhibiting the growth of a wide spectrum of microorganisms such as Gram-positive or negative bacteria, yeast, fungi, or parasites [4] It can also block pathogenic bacterial cells adhesion sites and allow the entry of entry of certain nutrients in gut lumen. It also facilitates the regulation of energy and modulation of the immune responses against antibodies [5] (Figure1).

Along with it, $L$. reuteri may be able to modulate the immune system in the gastrointestinal tract by stimulation of T-helper cells. This significant increase in T-helper cells may be a central mechanism of symbiosis for improving the health of the host gut and a key mechanism of action for this probiotic [6] (Figure2).

Clinical trials and a meta-analysis showed that Lactobacillus reuteri provides a reduction in daily crying time in patients with infantile colic. Also providing Lactobacillus reuteri in the diet can reduce the frequency of gastrointestinal symptoms like regurgitation, constipation and diarrhea in the first months of life [7-16]. 


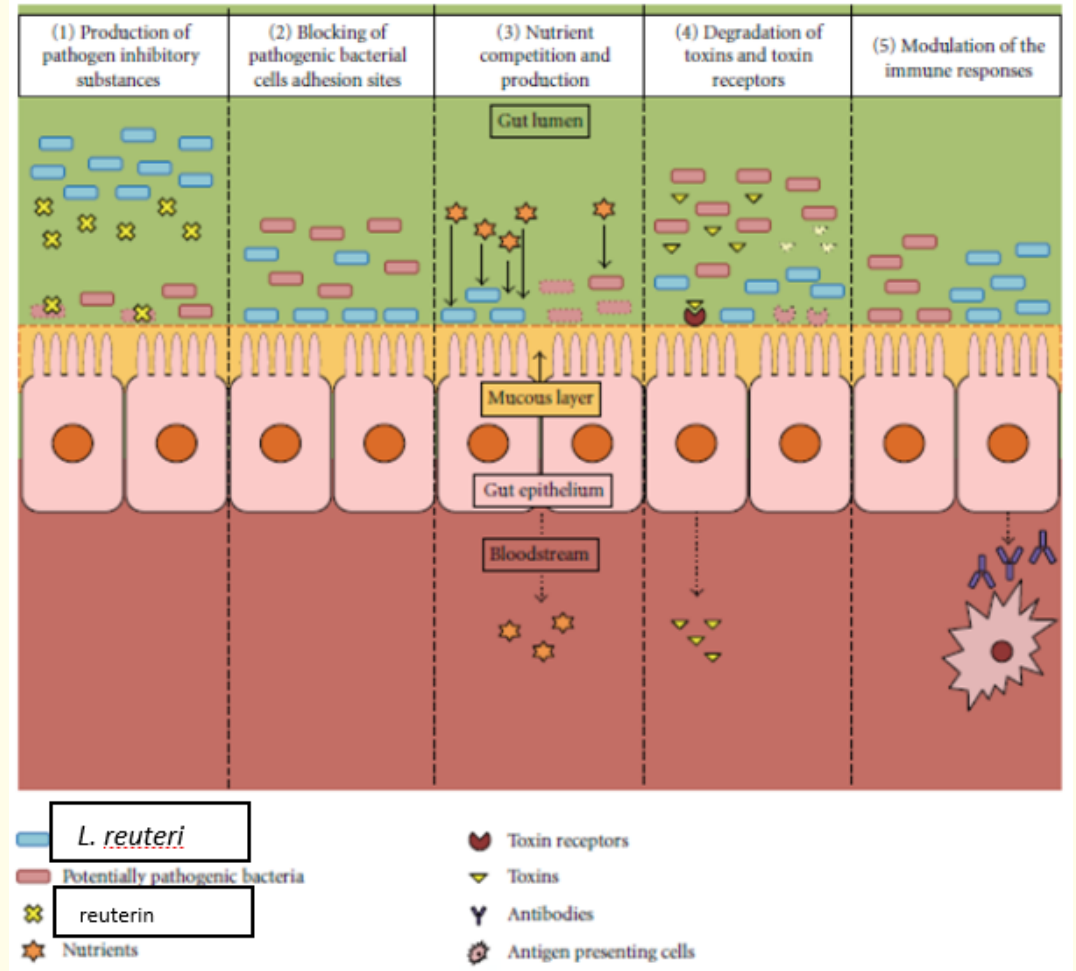

Figure 1: Pathways by which a probiotic can positively influence human health [5].

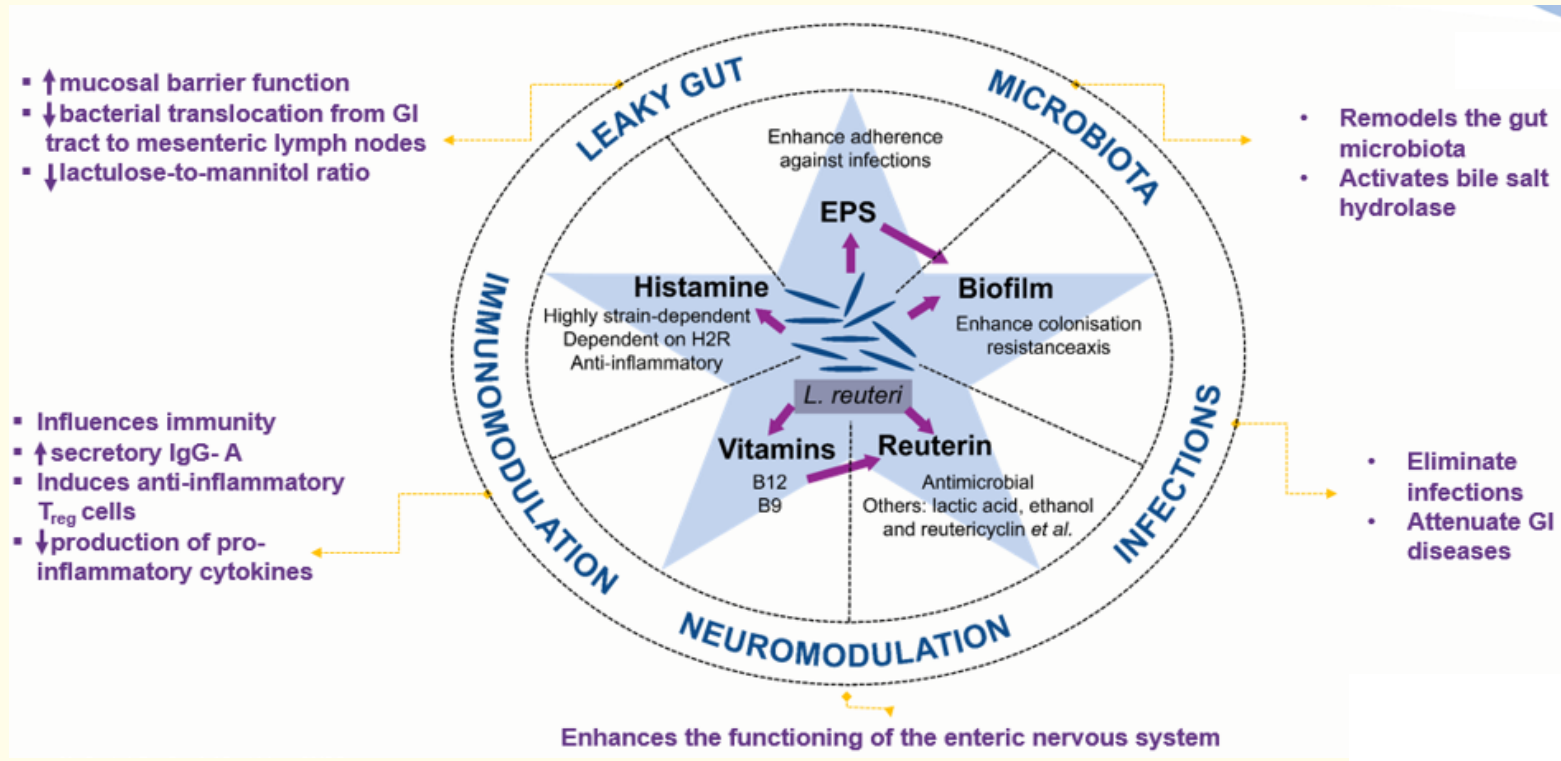

Figure 2: Pathways by which a probiotic can positively influence human health [5]. 


\section{Safety and Efficacy of L. reuteri}

Lactobacillus reuteri is one of the few endogenous Lactobacillus species in the human gastrointestinal tract, which has been used safely for many years as a probiotic dietary supplement in adults, and recent data demonstrate the safety after long-term dietary supplementation for newborn infants [6].

L. reuteri DSM 17938 at a dose of 108 colony-forming units per day in early breastfed infants improved symptoms of infantile colic and was well tolerated and safe [6] It is recognized safe by FDA's GRAS Notice Inventory Database pertaining to probiotic use in infant formula. "No adverse event reported for Lactobacillus reuteri DSM 17938 intended to use as an ingredient in term infant formula with an additional level of $10^{\wedge} 8-10^{\wedge} 10 \mathrm{CFU} / \mathrm{d}$ " [8].
Clinical evidences of $L$. reuteri helps in prevention and management FGIDs

Administration of $L$. reuteri has been proved to give various benefits in the management and prevention of gastrointestinal disorders like infantile colic, Functional Constipation, Diarrhea [10].

\section{L. reuteri prevents FGIDs}

A prospective, multicenter, double-masked, placebo-controlled randomized clinical trial suggest that oral supplementation with Lactobacillus reuteri DSM 17938 delivered at a dose of $10^{8} \mathrm{CFU}$ during the first 3 months of life can reduce the onset of colic, gastroesophageal reflux, and constipation in term newborns [10].
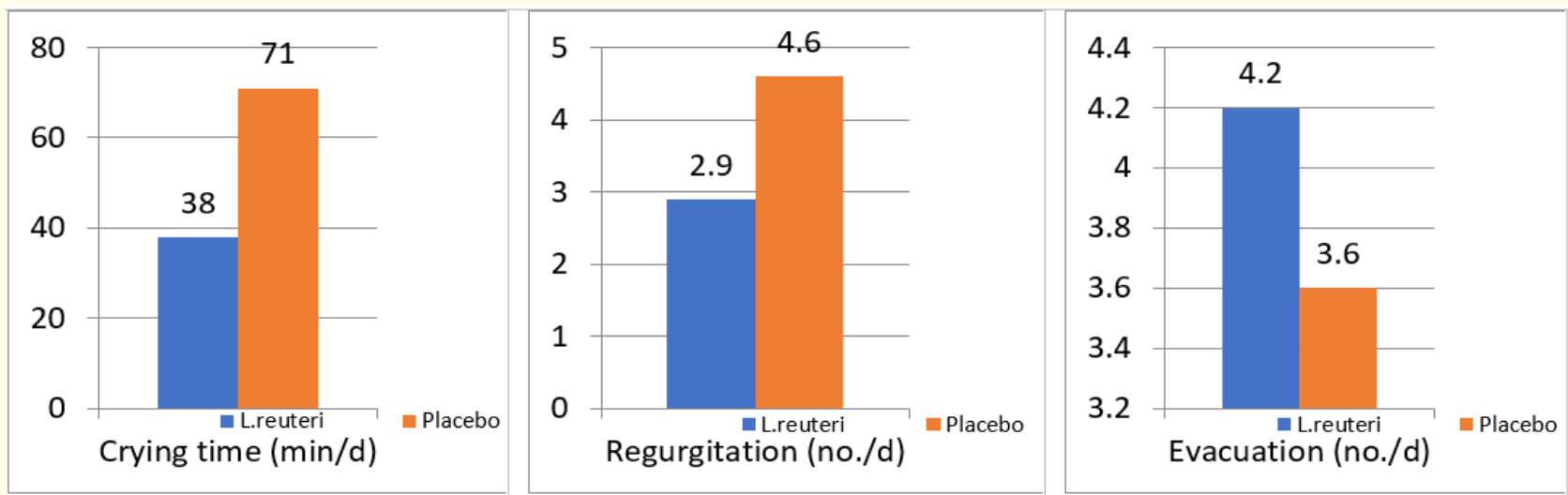

Figure 3: Impact of L. reuteri administration on regurgitation, colic and constipation.

\section{L. reuteri for the management of colic}

As many gastrointestinal disorders occur in infancy, which may be linked to several anatomical and functional changes observed in the first two years of life [9] Infantile colic is a common disturbance occurring in the first three months of life, which is a benign condition and one of the main causes of pediatric consultation because of its great impact on family life [10] Infantile colic occurs both in breastfed and formula fed infants equally.

Poor activity, swollen abdomen, excessive crying are the symptoms associated with colic. Gastroesophageal reflux, cow milk protein allergy can be some organic reasons for colic. Infect some suggest that swallowed air as a contributory factor but the colic pathophysiology is not well understood [7].
A randomized- double blind placebo control trial suggests that providing L. reuteri DSM 17938 to exclusively breastfed infants diagnosed with infantile colic proved superior to placebo in reducing daily crying and fussing times $[11,12]$.

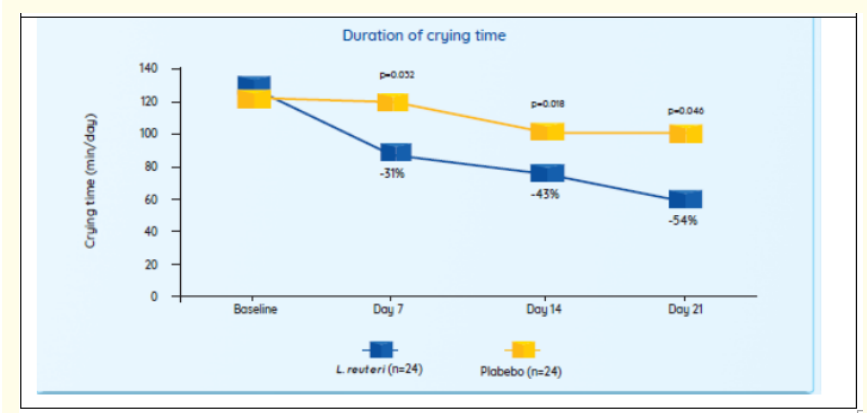

Figure 4: Proven to reduce 54\% of crying time [11]. 


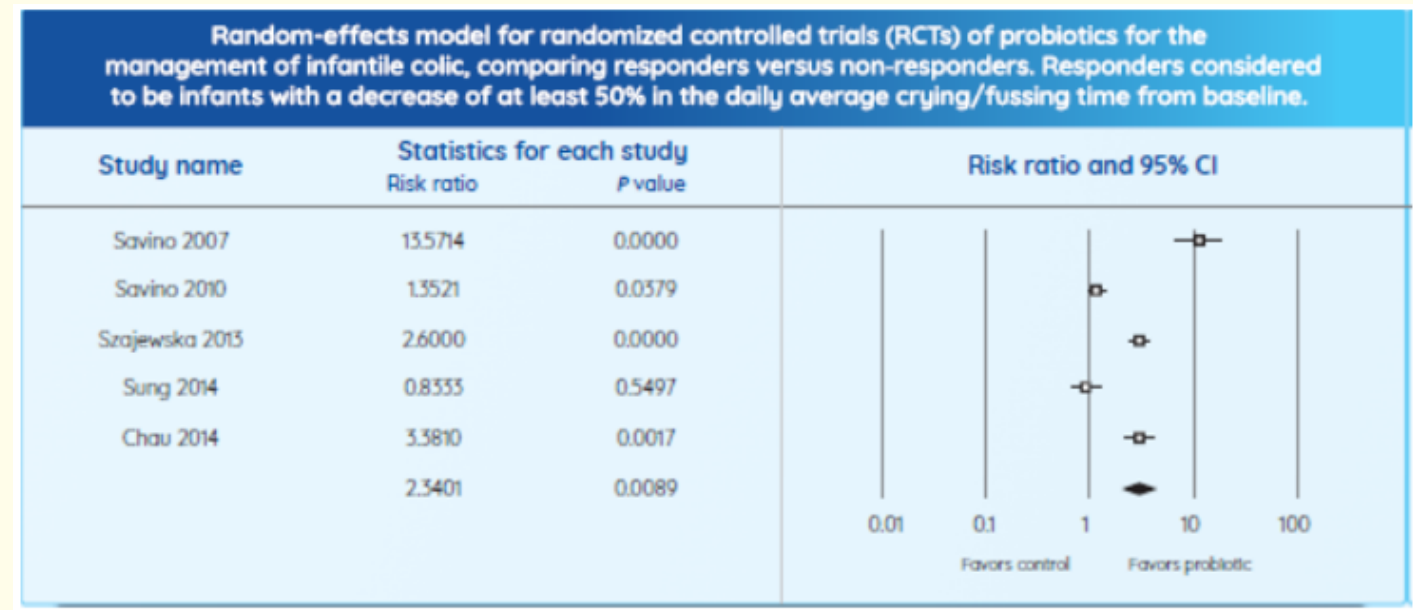

Figure 5

Multiple functional gastrointestinal disorders are more likely to be associated with affected quality of life leading to depression and irritation in mothers along with sleep issues, dietary issues and less comforting parent child relationship for both child and the mother than single functional gastrointestinal disorders [13]. Effective treatment of infantile colic with $L$. reuteri significantly improves quality of life for parents and families [13].

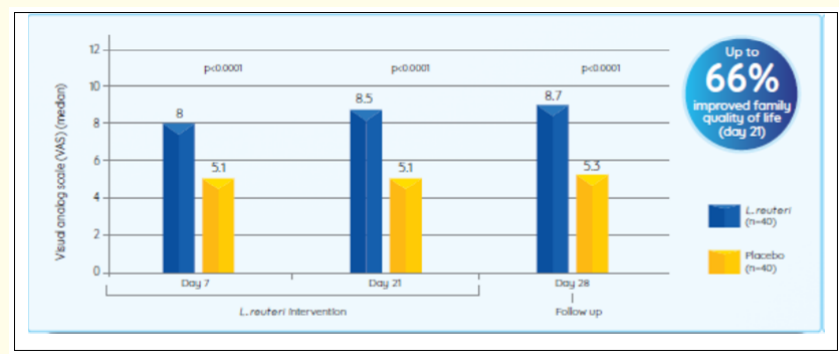

Figure 6: Impact of L. reuteri administration on family quality of life [13].

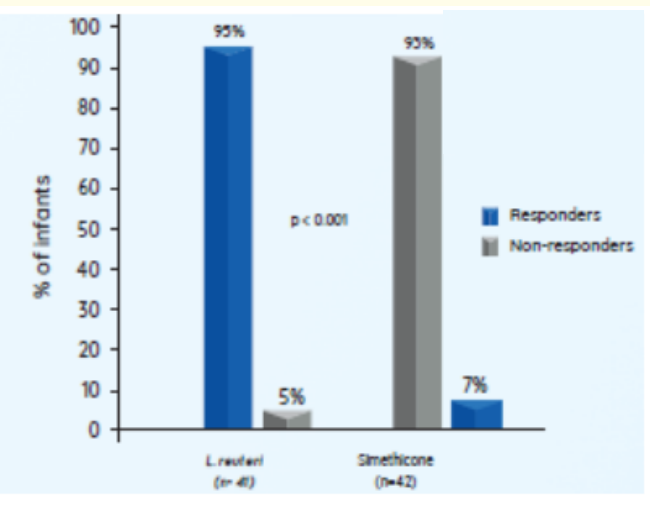

Figure 7: Response rate of $L$. reuteri vs Simethicone (most commonly prescribed medicine for infantile colic) [6].
Clinical evidences of $L$. reuteri helps in management of FGIDs other than colic

\section{L. reuteri significantly reduces frequency of regurgitation}

Functional gastroesophageal reflux (GER) is very common during infancy and most often manifests itself as episodes of regurgitation or vomiting. Lactobacillus reuteri administered to formula-fed preterm infants can results in a significantly increased gastric emptying rate and in a significant decrease in regurgitation [14].

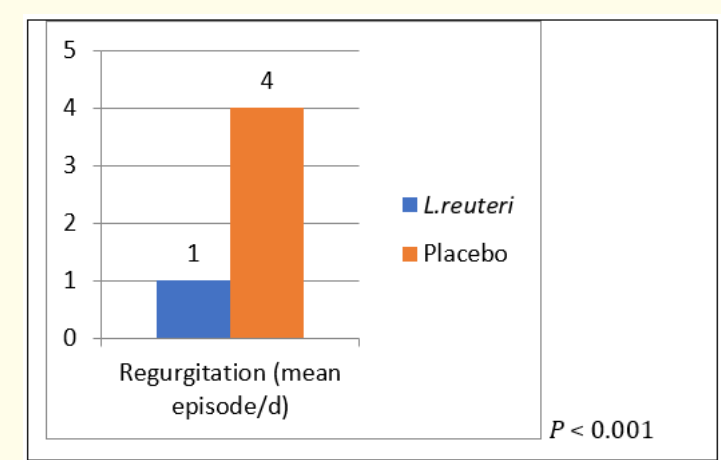

Figure 8: L. reuteri administration show a positive reduction in regurgitation [14].

Positive effect of $L$. reuteri in functional constipation

The administration of L. reuteri in infants with chronic constipation had a positive effect on bowel frequency. Evidence from a double-blind, randomized, placebo-controlled study conducted on 44 infants $>6$ months old with chronic constipation (22 assigned randomly to treatment with L. reuteri (DSM 17938), and 22 assigned to receive a placebo) suggest that infants treated with $L$. reuteri had a significantly higher defecation frequency or bowl movements than infants in the placebo group at week 2 of treatment [15]. 


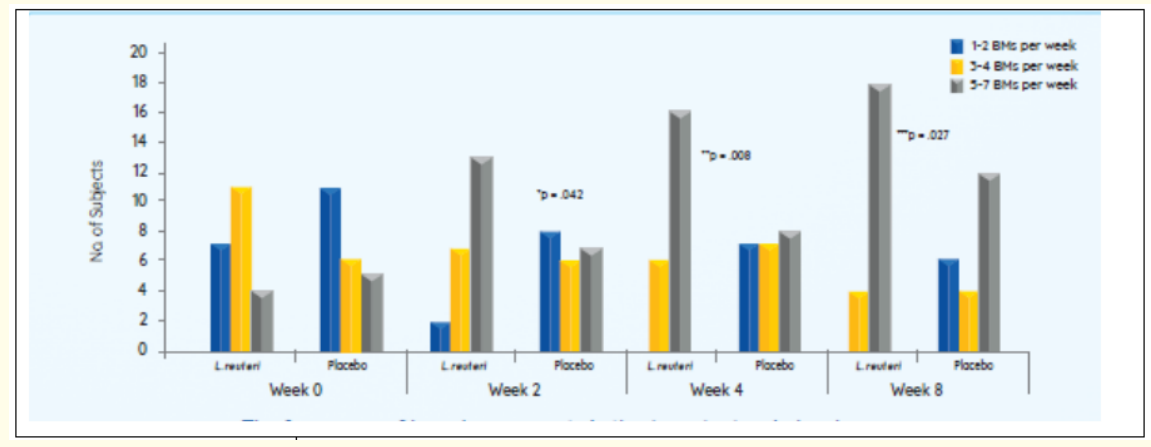

Figure 9: The frequency of bowel movements in the L. reuteri and placebo group [13].

Therapeutic effect of $L$ reuteri on infant diarrhea

The therapeutic effect of $L$. reuteri on infant diarrhoea has also been supported by several studies. The frequency of watery diarrhoea per 24-h period was significantly reduced on the second day of intervention in L. reuteri group compared to placebo [16].

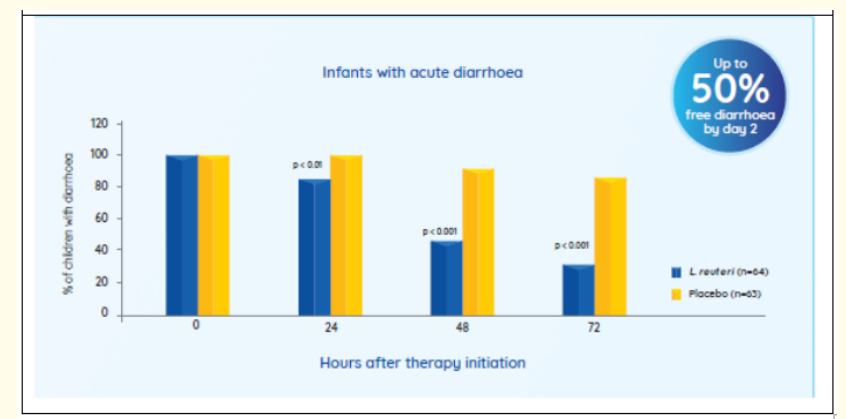

Figure 10

Administration of L. reuteri DSM 17938 significantly accelerates gastric emptying and ameliorated regurgitation in infants affected by non-complicated gastroesophageal reflux when used daily for 28 days with a dose of $1 \times 10^{8}$ (CFU) [4].

Several studies have well documented the safety and tolerance of infant formulas containing probiotics. Moreover, the data from various studies suggest that infants receiving $L$. reuteri in a starter infant formula showed age appropriate growth up to 6 months of age. They also had more soft stools showing that infant formulas supplemented with probiotics $L$. reuteri are safe, well tolerated, and support normal infant growth in an Indian population.

\section{Bibliography}

1. Palma GD., et al. "The microbiota-gut-brain axis in functional gastrointestinal disorders". Gut Microbes 5 (2014):3 419-429.

2. Benninga MA., et al. "Childhood Functional Gastrointestinal Disorders:Neonate/Toddler”. Gastroenterology 150 (2016): 1443-1455.

3. Sinkiewicz G and Ljunggren L. "Occurrence of Lactobacillus reuteri in human breast milk". Microbial Ecology in Health and Disease 20 (2008):122-126.

4. Urbańska M and Szajewska H. "The efficacy of Lactobacillus reuteri DSM 17938 in infants and children: a review of the current evidence". European Journal of Pediatrics 173.10 (2014): 1327-1337.

5. Prakash S., et al. "The Gut Microbiota and Human Health with an Emphasis on the Use of Microencapsulated Bacterial Cells". Journal of Biomedicine and Biotechnology (2011).

6. Valeur., et al. "Colonization and Immunomodulation by Lactobacillus reuteri ATCC 55730 in the Human Gastrointestinal Tract". Applied and Environmental Microbiology 70.2 (2004):1176-1181.

7. Savino F., et al. "Lactobacillus reuteri (American Type Culture Collection Strain 55730) Versus Simethicone in the Treatment of Infantile Colic: A Prospective Randomized Study". Pediatrics 119.1 (2007): e124.

8. Daelemans S., et al. "Recent advances in understanding and managing infantile colic". F100 Res 7 (2018): 1426. 
9. Notice to US Food and Drug Administration of the Conclusion that Inactivated Bacillus coagulans GBl-30, 6086 is Generally Recognized as Safe for use in Non-exempt Term Infant Formula.

10. Morais MB. "Sign and symptoms associated with digestive treatment of digestive tract development". Jornal De Pediatria 92.3 (2016): S46-S56.

11. Indrio F., et al. "Prophylactic Use of a Probiotic in the Prevention of Colic, Regurgitation, and Functional Constipation: A Randomized Clinical Trial". JAMA Pediatr (2013): 4367.

12. Chau K., et al. "Probiotics for Infantile Colic: A Randomized, Double-Blind, Placebo-Controlled Trial Investigating Lactobacillus reuteri DSM 17938". Journal of Pediatrics (2014).

13. Bird AS., et al. "Probiotics for the Treatment of Infantile Colic: A Systematic Review". Journal of Pharmacy Practice 30.3 (2017): 366-374.

14. BellaicheM., et al. "Multiple functional gastrointestinal disorders are frequent in formula-fed infants and decrease their quality of life". Acta Pædiatrica 107.7 (2018): 1276-1282.

15. Indrlo F, et al. "Prophylactic Use of a Probiotic in the Prevention of Colic, Regurgitation, and Functional Constipation A Randomized Clinical Trial". European Journal of Clinical Investigation 41.4 (2011): 417-422.

16. Coccorullo P., et al. "Lactobacillus reuteri (DSM 17938) in Infants with Functional Chronic Constipation: A Double-Blind, Randomized, Placebo-Controlled Study". Journal of Pediatrics 157.4 (2010): 598-602.

17. Dinleyici EC., et al. "Lactobacillus reuteri DSM 17938 effectively reduces the duration of acute diarrhoea in hospitalised children". Acta Paediatrica 103.7 (2014): e300-e305.

Volume 3 Issue 10 October 2019

(C) All rights are reserved by Ridhima Bhalla. 